\title{
MAXIMAL MULTIPLICATIVE SPATIAL-SPECTRAL CONCENTRATION ON THE SPHERE: OPTIMAL BASIS
}

\author{
Zubair Khalid and Rodney A. Kennedy \\ Research School of Engineering, The Australian National University, Canberra, ACT 2601, Australia \\ Email: \{zubair.khalid, rodney.kennedy\}@anu.edu.au
}

\begin{abstract}
In this work, we design complete orthonormal basis functions, which are referred to as optimal basis functions, that span the vector sum of subspaces formed by band-limited spatially concentrated and space-limited spectrally concentrated functions. The optimal basis are shown to be a linear combination of band-limited functions with maximized energy concentration in some spatial region of interest and space-limited functions which maximize the energy concentration in some spectral region. The linear combination is designed with an optimality condition of maximizing the product of measures of energy concentration in the spatial and spectral domain. We also show that each optimal basis is an eigenfunction of a linear operator which maximizes the product of energy concentration measures in spatial and spectral domain. Finally, we discuss the properties of the proposed optimal basis functions and highlight their usefulness for the signal representation and data analysis due to the simultaneous concentration of the proposed basis functions in spatial and spectral domains.
\end{abstract}

Index Terms - energy concentration problem; localized spectral analysis; spatial-spectral concentration; unit sphere.

\section{INTRODUCTION}

The development of spherical signal processing techniques has been an area of research and exploration in order to cater the needs of applications in various branches of science and engineering (e.g., [1$3]$ ), where the signals (finite energy functions) are inherently defined on the sphere. In most of these signal processing techniques [1,4-6], the signal is either analysed in spatial domain (sphere) or spectral domain. The spectral domain is characterized by spherical harmonic basis functions, which serve as natural basis functions for signals defined on the sphere [1]. For the representation of signals which have energy concentration in some spatial or spectral region or the reconstruction of a signal from its incomplete measurements, the spherical harmonic basis may not be an optimal choice $[3,7-10]$. In this paper, we consider the problem of designing basis functions which are simultaneously concentrated in spatial and spectral domain.

\subsection{Relation to Prior Work}

It is well-known that functions cannot have finite support in both the spatial (or time) and spectral (or frequency) domain at the same time $[3,11]$. The Slepian concentration problem [11-13], initially formulated in the Euclidean domain, has been recently investigated for signals defined on the sphere to obtain the band-limited (or spacelimited) basis functions with optimal concentration in some spatial (or spectral) region of interest [3,7]. The orthogonal family

This work was supported under the Australian Research Council's Discovery Projects funding scheme (Project no. DP150101011). of functions, referred as Slepian basis, arise from the solution of Slepian concentration problem on the sphere have been used in various signal processing applications $[4-6,8,9]$. The Slepian basis are localized in one domain (spatial or spectral) and concentrated in the other domain and therefore becomes an attractive choice for the representation and analysis of spatially (or spectrally) concentrated band-limited (or space-limited) signal [3].

In addition to the Slepian concentration problem on the sphere, a quadratic variational framework, originally formulated by Franks for time-frequency signals, has been developed in [14] for signals on the sphere, which maximizes the sum of arbitrarily weighted spatial and spectral energy concentration measures, and therefore generalizes the Slepian concentration problem. For signals on the sphere, the energy concentration problems, explored in the literature, either maximize the measure of energy concentration in spatial or spectral domain, or maximize the weighted sum of measures of energy concentration in spatial and spectral domain. However, the problem of maximizing the product of energy concentrations measures, investigated for signals defined on one dimensional Euclidean domain (time) in [15], has not been considered for signals on the sphere.

\subsection{Contributions}

In this work, we seek an orthogonal family of functions which are maximally concentrated in both the spatial and spectral domain. We consider the design of basis functions for the subspace, referred as joint subspace, given by the vector sum of two subspaces formed by band-limited functions and space-limited spectrally concentrated functions. We construct a class of functions for the joint subspace as a linear combination of the band-limited functions and space-limited functions, which arise from the solution of Slepian concentration problems on the sphere. We optimise the linear combination such that the product of measures of energy concentration in spatial and spectral domain is maximized. We later show that the constructed set of functions forms a complete orthonormal basis, referred as optimal basis, for the joint subspace. Furthermore, we present the properties exhibited by the optimal basis functions. In order to quantify the energy concentration in the spatial and spectral regions of interest, we also formulate a linear operator which maximizes the product of energy concentration measures and show that the each optimal basis function is an eigenfunction of the operator.

We organize the rest of the paper as follows. We review the mathematical background and Slepian concentration problem on the sphere in Section 2 and Section 3 respectively. In Section 4, we construct a set of functions which serves as optimal basis for the joint subspace. The concluding remarks are then made in Section 5. 


\section{SIGNALS ON THE SPHERE}

We consider the complex-valued square-integrable functions defined on the sphere, denoted by $\mathbb{S}^{2}$. These functions form a Hilbert space denoted by $L^{2}\left(\mathbb{S}^{2}\right)$ equipped with the following inner product for two functions $f, h \in L^{2}\left(\mathbb{S}^{2}\right)$,

$$
\langle f, h\rangle \triangleq \int_{\mathbb{S}^{2}} f(\widehat{\boldsymbol{x}}) \overline{h(\widehat{\boldsymbol{x}})} d s(\widehat{\boldsymbol{x}}),
$$

where $\widehat{\boldsymbol{x}} \equiv \widehat{\boldsymbol{x}}(\theta, \phi) \triangleq(\sin \theta \cos \phi, \sin \theta \sin \phi, \cos \theta)^{T} \in \mathbb{R}^{3}$ is a unit vector that parameterizes a point on the unit sphere with $\theta \in[0, \pi]$ denoting the co-latitude and $\phi \in[0,2 \pi)$ denoting the longitude, $\overline{(\cdot)}$ denotes the complex conjugate operation, $d s(\widehat{\boldsymbol{x}})=\sin \theta d \theta d \phi$ is the differential area element on $\mathbb{S}^{2}$ and the integration is carried out over $\mathbb{S}^{2}$. The inner product induces a norm $\|f\| \triangleq\langle f, f\rangle^{1 / 2}$. We refer the functions with finite energy (finite induced norm) as "signals on the sphere". Also define $\langle f, g\rangle_{R} \triangleq \int_{R} f(\widehat{\boldsymbol{x}}) \overline{g(\widehat{\boldsymbol{x}})} d s(\widehat{\boldsymbol{x}})$ for any region $R \subset \mathbb{S}^{2}$

\subsection{Spherical Harmonics and Spectral Domain Representation}

Spherical harmonics, denoted by $Y_{\ell}^{m}(\theta, \phi)$ for integer degree $\ell \geq 0$ and integer order $m \in[-\ell, \ell]$ form complete orthonormal set of basis functions for $L^{2}\left(\mathbb{S}^{2}\right)$, and therefore we can expand any signal $f \in L^{2}\left(\mathbb{S}^{2}\right)$ as

$$
f(\widehat{\boldsymbol{x}})=\sum_{\ell=0}^{\infty} \sum_{m=-\ell}^{\ell}(f)_{\ell}^{m} Y_{\ell}^{m}(\widehat{\boldsymbol{x}}), \quad(f)_{\ell}^{m} \triangleq\left\langle f, Y_{\ell}^{m}\right\rangle,
$$

where $(f)_{\ell}^{m}$ denotes the spherical harmonic coefficient of degree $\ell$ and order $m$. The spherical harmonic coefficients $(f)_{\ell}^{m}$ form the spectral domain representation of the signal.

\subsection{Important Subspaces of $L^{2}\left(\mathbb{S}^{2}\right)$}

The signal $f$ is said to be band-limited within the spectral region $A_{L} \triangleq\{0 \leq \ell \leq L-1,|m| \leq \ell\}$ if $(f)_{\ell}^{m}=0, \forall \ell>L$. The set of such band-limited functions form an $L^{2}$ dimensional space $\mathcal{H}_{L}$ as a subspace of $L^{2}\left(\mathbb{S}^{2}\right)$. Also define $\mathcal{H}_{R}$ as the space of finite energy space-limited functions confined within the region $R \subset \mathbb{S}^{2} . \mathcal{H}_{R}$ is an infinite dimensional subspace of $L^{2}\left(\mathbb{S}^{2}\right)$.

\subsection{Important Operators on the Sphere}

For signals on the sphere, we define a linear integral operator $\mathcal{S}$ defined by its kernel $S(\widehat{\boldsymbol{x}}, \widehat{\boldsymbol{y}})$ using Fredholm integral equation, as [1]

$$
(\mathcal{S} f)(\widehat{\boldsymbol{x}})=\int_{\mathbb{S}^{2}} S(\widehat{\boldsymbol{x}}, \widehat{\boldsymbol{y}}) f(\widehat{\boldsymbol{y}}) d s(\widehat{\boldsymbol{y}}),
$$

where $S(\widehat{\boldsymbol{x}}, \widehat{\boldsymbol{y}})$ is the kernel for an operator $\mathcal{S}$.

Spatial Selection Operator : Define the spatial selection operator $\mathcal{S}_{R}$ that selects the function in a region $R \subset \mathbb{S}^{2}$ with kernel given by

$$
S_{R}(\widehat{\boldsymbol{x}}, \widehat{\boldsymbol{y}}) \triangleq I_{R}(\widehat{\boldsymbol{x}}) \delta(\widehat{\boldsymbol{x}}, \widehat{\boldsymbol{y}}),
$$

where $I_{R}(\widehat{\boldsymbol{x}})=1$ for $\widehat{\boldsymbol{x}} \in R \subset \mathbb{S}^{2}$ and $I_{R}(\widehat{\boldsymbol{x}})=0$ for $\widehat{\boldsymbol{x}} \in \mathbb{S}^{2} \backslash R$ is an indicator function of the region $R$ and $\delta(\widehat{\boldsymbol{x}}, \widehat{\boldsymbol{y}})$ denotes the Dirac delta function on the sphere.

Spectral Selection Operator: Define the spectral selection operator $\overline{\mathcal{S}}_{L}$, which band-limits the signal within the spectral region $A_{L}$, by its kernel

$$
S_{L}(\widehat{\boldsymbol{x}}, \widehat{\boldsymbol{y}}) \triangleq \sum_{\ell=0}^{L-1} \sum_{m=-\ell}^{\ell} Y_{\ell}^{m}(\widehat{\boldsymbol{x}}) \overline{Y_{\ell}^{m}(\widehat{\boldsymbol{y}})}
$$

which is also referred to as band-limited Dirac delta function [1,3].

The selection operators $\mathcal{S}_{R}$ and $\mathcal{S}_{L}$ project the signal $f \in$ $L^{2}\left(\mathbb{S}^{2}\right)$ onto the subspaces $\mathcal{H}_{R}$ and $\mathcal{H}_{L}$, respectively. Using the selection operators, we also define $\|f\|_{R}^{2} \triangleq\left\langle\mathcal{S}_{R} f, \mathcal{S}_{R} f\right\rangle=\langle f, f\rangle_{R}$ and $\|f\|_{L}^{2} \triangleq\left\langle\mathcal{S}_{L} f, \mathcal{S}_{L} f\right\rangle$, which respectively quantify the energy concentration of the signal $f \in L^{2}\left(\mathbb{S}^{2}\right)$ in the spatial region $R$ and the spectral region $A_{L}$.

\section{SPATIAL-SPECTRAL CONCENTRATION ON THE SPHERE AND PROBLEM FORMULATION}

In this section, we review the Slepian concentration problem on the sphere of finding the band-limited functions with maximum energy concentration in the spatial region or the space-limited functions with maximum energy concentration in the spectral region $[3,7,8]$.

The problem of maximizing the spatial concentration of unit energy band-limited function $f \in \mathcal{H}_{L}$ within the spatial region $R \subset \mathbb{S}^{2}$ can be formulated as a Fredholm integral equation, given by [3]

$$
\left(\mathcal{S}_{L} \mathcal{S}_{R} f\right)(\widehat{\boldsymbol{x}})=\lambda f(\widehat{\boldsymbol{x}}), \quad \widehat{\boldsymbol{x}} \in \mathbb{S}^{2},
$$

or equivalently, using (4), (5) and (2), as an algebraic eigenvalue problem of size $L^{2}$, given by

$\sum_{\ell^{\prime}=0}^{L-1} \sum_{m^{\prime}=-\ell^{\prime}}^{\ell^{\prime}}(f)_{\ell^{\prime}}^{m^{\prime}} \int_{R} Y_{\ell^{\prime}}^{m^{\prime}}(\widehat{\boldsymbol{x}}) \overline{Y_{\ell}^{m}(\widehat{\boldsymbol{x}})} d s(\widehat{\boldsymbol{x}})=\lambda(f)_{\ell}^{m}, \quad \ell, m \in A_{L}$,

the solution of which provides $L^{2}$ band-limited eigenfunctions. Let the eigenfunctions be denoted by $f_{p}$ for $p \in\left[1, L^{2}\right]$. The eigenvalue $0<\lambda_{p}<1$ associated with each band-limited eigenfunction $f_{p}$ quantifies the energy concentration of the eigenfunction in the region $R$. The eigenfunctions are indexed such that $1>\lambda_{1} \geq \lambda_{2} \geq \ldots \geq$ $\lambda_{L^{2}}>0$.

For the maximization of the spectral concentration of unit energy space-limited function $h \in \mathcal{H}_{R}$ within the spectral region $A_{L}$, an eigenvalue problem, similar to (6), is given by [3]

$$
\left(\mathcal{S}_{R} \mathcal{S}_{L} h\right)(\widehat{\boldsymbol{x}})=\lambda h(\widehat{\boldsymbol{x}}), \quad \widehat{\boldsymbol{x}} \in R .
$$

The Fredholm integral equations in (6) and (7) are exactly same for $\widehat{\boldsymbol{x}} \in R$ [3], therefore the band-limited eigenfunctions of (6) are identical to the space-limited eigenfunctions of (7) within the region $R$. Each band-limited eigenfunction $f_{p} \in \mathcal{H}_{L}$ gives rise to a spacelimited eigenfunction $h_{p}=\mathcal{S}_{R} f_{p} \in \mathcal{H}_{R}$ for $p \in\left[1, L^{2}\right]$. The eigenvalue $\lambda_{p}$ serves as a measure of the energy concentration of the space-limited function $h_{p}$ in the spectral region $A_{p}$.

The band-limited and space-limited eigenfunctions follow the following orthogonality relations [3]

$$
\begin{aligned}
& \left\langle f_{p}, f_{q}\right\rangle=\delta_{p, q}=\left\|f_{p}\right\|_{L}^{2}, \quad\left\|f_{p}\right\|_{R}^{2}=\left\langle f_{p}, f_{q}\right\rangle_{R}=\delta_{p, q} \lambda_{p}, \\
& \left\|h_{p}\right\|_{R}^{2}=\left\langle h_{p}, h_{q}\right\rangle=\left\langle h_{p}, h_{q}\right\rangle_{R}=\delta_{p, q} \lambda_{p}, \quad\left\|h_{p}\right\|_{L}^{2}=\lambda_{p}^{2},
\end{aligned}
$$

for $p, q \in\left[1, L^{2}\right]$, where $\delta_{p, q}$ is the Kronecker delta.

\subsection{Problem Under Consideration}

The set of band-limited eigenfunctions $f_{p} \in \mathcal{H}_{L}, p \in\left[1, L^{2}\right]$ forms an orthonormal basis, referred as Slepian band-limited basis, for the subspace $\mathcal{H}_{L}$. Furthermore, space-limited eigenfunctions $h_{p} \in \mathcal{H}_{R}$, $p \in\left[1, L^{2}\right]$ serve as orthogonal basis functions for the representation of any space-limited function within the spectral region $A_{L}$. Let the subspace spanned by the space-limited eigenfunctions be denoted by $\widetilde{\mathcal{H}}_{R} \subset \mathcal{H}_{R} \subset L^{2}\left(\mathbb{S}^{2}\right)$. For the representation of a signal, the use of band-limited or space-limited eigenfunctions becomes an attractive choice if the band-limited signal is concentrated in some spatial region $R$ or the space-limited signal is concentrated 
in some spectral region $A_{L}$, respectively, as such signals can be represented using fewer number of eigenfunctions, in comparison to their representation in the spherical harmonic basis functions [3]. In practice, the signal may not be completely band-limited nor spacelimited. Therefore the functions which maximize energy concentration in both the spatial and spectral domain become a more suitable choice for the representation of signal that is neither band-limited, nor space-limited, but, concentrated (simultaneously) in both the spatial and spectral domains. In this work, we develop basis functions which maximize the energy concentration, simultaneously, in both the spatial and spectral domains.

\section{DESIGN OF OPTIMAL BASIS}

We develop basis functions, which span the subspace $\mathcal{H}_{L}+\widetilde{\mathcal{H}}_{R}$, the vector sum of $\mathcal{H}_{L}$ and $\widetilde{\mathcal{H}}_{R}$, and maximize the product of the energy concentration in the spatial region $R$ and the energy concentration in the spectral region $A_{L}$. We refer to the subspace $\mathcal{H}_{L}+\widetilde{\mathcal{H}}_{R}$ as joint subspace. Any signal $z \in \mathcal{H}_{L}+\widetilde{\mathcal{H}}_{R}$ can be written as $z(\widehat{\boldsymbol{x}})=$ $z_{L}(\widehat{\boldsymbol{x}})+z_{R}(\widehat{\boldsymbol{x}})$, where $z_{L} \in \mathcal{H}_{L}$ and $z_{R} \in \widetilde{\mathcal{H}}_{R}$. Since the bandlimited eigenfunctions $f_{p}, p \in\left[1, L^{2}\right]$ are maximally concentrated in the spatial region $R$, while the space-limited eigenfunctions $h_{p}=$ $\mathcal{S}_{R} f_{p}, p \in\left[1, L^{2}\right]$ are maximally concentrated in the spectral region $A_{L}$, we construct a class of functions as linear combinations of the space-limited and band-limited bases, as follows:

$$
g_{p}(\widehat{\boldsymbol{x}}) \triangleq \alpha_{p} f_{p}(\widehat{\boldsymbol{x}})+\beta_{p} h_{p}(\widehat{\boldsymbol{x}}), \quad h_{p}(\widehat{\boldsymbol{x}})=\left(\mathcal{S}_{R} f_{p}\right)(\widehat{\boldsymbol{x}}),
$$

for $p \in\left[1, L^{2}\right]$, where we optimally choose $\alpha_{p}$ and $\beta_{p}$ such that the product of the measures of the energy concentration of the function $g_{p}$ in the spatial region $R$ and in the spectral region $A_{L}$ is maximized with respect to unit energy constraint on $g_{p}$. Since $f_{p} \in \mathcal{H}_{L}$ and $h_{p} \in \widetilde{\mathcal{H}}_{R}$, we note that every $g_{p} \in \mathcal{H}_{L}+\widetilde{\mathcal{H}}_{R}$. We determine the weights $\alpha_{p}$ and $\beta_{p}$ in the following theorem.

Theorem 1. For function $g_{p} \in \mathcal{H}_{L}+\widetilde{\mathcal{H}}_{R}$, formulated in (9), the product of the energy concentration in the spatial region $R \subset \mathbb{S}^{2}$, given by $\left\|g_{p}\right\|_{R}^{2}$, and the energy concentration in the spectral region $A_{L}$, given by $\left\|g_{p}\right\|_{L}^{2}$ is maximized with respect to the unit energy constraint on $g_{p}$ for the following two pairs of $\alpha_{p}$ and $\beta_{p}$

$$
\alpha_{p, 1}=F, \quad \beta_{p, 1}=\frac{F}{\sqrt{\lambda_{p}}}, \quad \alpha_{p, 2}=G, \quad \beta_{p, 2}=-\frac{G}{\sqrt{\lambda_{p}}},
$$

where $F=\left(2+2 \sqrt{\lambda_{p}}\right)^{-1 / 2}$ and $G=\left(2-2 \sqrt{\lambda_{p}}\right)^{-1 / 2}$.

Proof. Using the properties of band-limited and space-limited bases (eigenfunctions), given in (8), the spatial energy concentration and the spectral energy concentration can be formulated as

$$
\left\|g_{p}\right\|_{R}^{2}=\lambda_{p}\left(\alpha_{p}+\beta_{p}\right)^{2}, \quad\left\|g_{p}\right\|_{L}^{2}=\left(\alpha_{p}+\beta_{p} \lambda_{p}\right)^{2} .
$$

The maximization of the product of $\left\|g_{p}\right\|_{R}^{2}$ and $\left\|g_{p}\right\|_{L}^{2}$ with respect to the unit energy constraint $\left\|g_{p}\right\|^{2}=1$ yields the two solutions for $\alpha_{p}$ and $\beta_{p}$, given in (10).

Remark 1. Since we obtain two pairs of $\alpha_{p}$ and $\beta_{p}$, we define a set of functions, given by

$g_{p, k}(\widehat{\boldsymbol{x}})=\alpha_{p, k} f_{p}(\widehat{\boldsymbol{x}})+\beta_{p, k}\left(\mathcal{S}_{R} f_{p}\right)(\widehat{\boldsymbol{x}}), \quad p \in\left[1, L^{2}\right], k=1,2$,

with the measures of the energy concentration, given by,

$$
\left\|g_{p, k}\right\|_{R}^{2}=\left\|g_{p, k}\right\|_{L}^{2}=\frac{1-(-1)^{k} \sqrt{\lambda_{p}}}{2}, \quad k=1,2
$$

which are obtained by substituting the values for $\alpha_{p, k}$ and $\beta_{p, k}$, given in (10), in (11).
We now show that the functions given in (12) serve as complete basis for the joint subspace $\mathcal{H}_{L}+\widetilde{\mathcal{H}}_{R}$.

Theorem 2. The set of functions $g_{p, k}, p \in\left[1, L^{2}\right], k=1,2$, of the form given in (12), forms a complete orthonormal basis of the joint subspace $\mathcal{H}_{L}+\widetilde{\mathcal{H}}_{R}$ for $\alpha_{p, k}$ and $\beta_{p, k}$ given in (10).

Proof. Since $h_{p}=\mathcal{S}_{R} f_{p}$, (8) implies that $\left\langle h_{p}, f_{q}\right\rangle_{R}=\lambda_{p} \delta_{p, q}$, which together with the orthogonality of band-limited functions and space-limited functions, imply that the functions $g_{p, 1}$ and $g_{q, 2}$ for $p, q \in\left[1, L^{2}\right], p \neq q$ are orthogonal (in fact orthonormal due to the unit energy constraint on $g_{p, k}$ ) to each other for any values of $\alpha_{p, 1}, \alpha_{q, 2}, \beta_{p, 1}$ and $\beta_{q, 2}$. For the values of $\alpha_{p, k}$ and $\beta_{p, k}$ given in (10), it can be easily shown that $g_{p, 1}$ and $g_{p, 2}$ also become orthonormal for each $p \in\left[1, L^{2}\right]$. Since each of the subspaces $\widetilde{\mathcal{H}}_{R}$ and $\mathcal{H}_{L}$ is of dimension $L^{2}$, we note that $\mathcal{H}_{L}+\widetilde{\mathcal{H}}_{R}$ is $2 L^{2}$ dimensional subspace and therefore the $2 L^{2}$ number of orthonormal functions $g_{p, k} \in \mathcal{H}_{L}+\widetilde{\mathcal{H}}_{R}$ for $p \in\left[1, L^{2}\right]$, and $k=1,2$ form a complete basis for $\mathcal{H}_{L}+\widetilde{\mathcal{H}}_{R}$.

We now have a set of functions, $g_{p, k}, p \in\left[1, L^{2}\right], k=1,2$, which forms the basis for the joint subspace $\mathcal{H}_{L}+\widetilde{\mathcal{H}}_{R}$. Since these basis functions are designed with an optimality condition of maximizing the product of the measures of the energy concentration in the spatial region $R$ and spectral region $A_{L}$, we refer to these basis as optimal basis. Among the optimal basis functions, we are more interested in the functions that are highly concentrated in the spatial region $R$ and in the spectral region $A_{L}$. The energy concentration levels, given in (13), measure the energy of the signal spatial region $R$ and the spectral region $A_{L}$ and indicate that each $g_{p, k}$ attains equal energy concentration in both the spatial and spectral regions. However, these measures do not quantify the simultaneous concentration in these regions of interest. In order to derive some measure for the the simultaneous energy concentration, we develop a linear integral operator by formulating a Fredholm integral equation for the problem of maximizing the product of energy concentration levels in both the spatial domain and spectral domain with in the regions of interest. We then show that each of the basis functions $g_{p, k}$ is an eigenfunction of the operator and the eigenvalue associated with each eigenfunction serves as a measure of simultaneous concentration.

Theorem 3. The function $w \in L^{2}\left(\mathbb{S}^{2}\right)$ which maximizes the product of the energy concentration in the spatial region $R \subset \mathbb{S}^{2}$, given by $\|w\|_{R}^{2}$, and the energy concentration in the spectral region $A_{L}$, given by $\|w\|_{L}^{2}$, is an eigenfunction of an integral operator $\mathcal{S}_{R L}$ with kernel given by

$$
S_{R L}(\widehat{\boldsymbol{x}}, \widehat{\boldsymbol{y}})=\frac{I_{R}(\widehat{\boldsymbol{x}})+I_{R}(\widehat{\boldsymbol{y}})}{2} \sum_{\ell=0}^{L-1} \sum_{m=-\ell}^{\ell} Y_{\ell}^{m}(\widehat{\boldsymbol{x}}) \overline{Y_{\ell}^{m}(\widehat{\boldsymbol{y}})},
$$

and the eigenvalue, denoted by $\eta$, of the eigenfunction $w$ such that $\left(\mathcal{S}_{R L} w\right)(\widehat{\boldsymbol{x}})=\eta w(\widehat{\boldsymbol{x}})$ is given by

$$
\eta=\|w\|_{R}^{2}\left(2\|w\|_{R}^{2}-1\right)
$$

Proof. We apply the variational principle, also adopted in [15], to find the unit energy function $w$, that maximizes the product of the energy concentration levels in the spatial and spectral region. Let $W^{\epsilon}(\widehat{\boldsymbol{x}})=w(\widehat{\boldsymbol{x}})+\epsilon z(\widehat{\boldsymbol{x}})$, where $z \in L^{2}\left(\mathbb{S}^{2}\right)$ and $\epsilon \in \mathbb{R}$ is a small number that quantifies the perturbation in the solution. We find $w$ by maximizing the product of the energy concentration given by

$$
Q=\frac{\left\|W^{\epsilon}\right\|_{R}^{2}}{\left\|W^{\epsilon}\right\|^{2}} \frac{\left\|W^{\epsilon}\right\|_{L}^{2}}{\left\|W^{\epsilon}\right\|^{2}}
$$


where we have divided the energy concentration levels by $\left\|W^{\epsilon}\right\|^{2}$ for unit energy normalization. Since $0 \leq Q \leq 1$, we maximize $\log Q$ in order to maximize $Q$ as follows

$$
\left.\frac{d}{d \epsilon}(\log Q)\right|_{\epsilon=0}=\left.\frac{d}{d \epsilon}\left(\log \frac{\left\|W^{\epsilon}\right\|_{R}^{2}}{\left\|W^{\epsilon}\right\|^{2}}+\log \frac{\left\|W^{\epsilon}\right\|_{L}^{2}}{\left\|W^{\epsilon}\right\|^{2}}\right)\right|_{\epsilon=0}=0,
$$

which is simplified, by noting that $\left\|W^{\epsilon}\right\|_{R}=\left\langle\mathcal{S}_{R} W^{\epsilon}, \mathcal{S}_{R} W^{\epsilon}\right\rangle$ and $\left\|W^{\epsilon}\right\|_{L}=\left\langle\mathcal{S}_{L} W^{\epsilon}, \mathcal{S}_{L} W^{\epsilon}\right\rangle$, to obtain

$\frac{1}{2\|w\|_{R}^{2}}\left(\mathcal{S}_{R}(w z)\right)(\widehat{\boldsymbol{x}})+\frac{1}{2\|w\|_{L}^{2}}\left(\mathcal{S}_{L}(w z)\right)(\widehat{\boldsymbol{x}})-w(\widehat{\boldsymbol{x}}) z(\widehat{\boldsymbol{x}})=0$, which holds for every $z \in L^{2}\left(\mathbb{S}^{2}\right)$, therefore

$$
\frac{\|w\|_{L}^{2}}{2}\left(\mathcal{S}_{R} w\right)(\widehat{\boldsymbol{x}})+\frac{\|w\|_{R}^{2}}{2}\left(\mathcal{S}_{L} w\right)(\widehat{\boldsymbol{x}})-\|w\|_{R}^{2}\|w\|_{L}^{2} w(\widehat{\boldsymbol{x}})=0 .
$$

Now, we multiply (17) by $\overline{w(\widehat{\boldsymbol{x}})}$, apply the operator $\mathcal{S}_{R}$ and integrate over the sphere to obtain

$$
\frac{\|w\|_{L}^{2}}{2}+\frac{1}{2}\left\langle\mathcal{S}_{R} \mathcal{S}_{L} w, \mathcal{S}_{R} w\right\rangle-\|w\|_{R}^{2}\|g\|_{L}^{2}=0 .
$$

Again multiplying (17) by $\overline{w(\widehat{\boldsymbol{x}})}$, applying the operator $\mathcal{S}_{L}$ and integrating over the sphere yield

$$
\frac{1}{2}\left\langle\mathcal{S}_{L} \mathcal{S}_{R} w, \mathcal{S}_{L} w\right\rangle+\frac{\|w\|_{R}^{2}}{2}-\|w\|_{R}^{2}\|w\|_{L}^{2}=0
$$

Using the formulation of kernels for the operators $\mathcal{S}_{R}$ and $\mathcal{S}_{L}$, given in (4) and (5), respectively, and employing the orthonormality of spherical harmonics, it can be shown that

$$
\left\langle\mathcal{S}_{R} \mathcal{S}_{L} w, \mathcal{S}_{R} w\right\rangle=\overline{\left\langle\mathcal{S}_{L} \mathcal{S}_{R} w, \mathcal{S}_{L} w\right\rangle}=\sum_{\ell=0}^{L-1} \sum_{m=-\ell}^{\ell}(w)_{\ell}^{m}\left\langle Y_{\ell}^{m}, w\right\rangle_{R},
$$

which implies, through (18) and (19), that

$$
\|w\|_{R}^{2}=\|w\|_{L}^{2}
$$

which is used in the remainder of the proof. By applying the operator $\mathcal{S}_{L}$ on $(17)$, we get $\left(\mathcal{S}_{L} \mathcal{S}_{R}\right) w(\widehat{\boldsymbol{x}})=\left(2\|w\|_{R}^{2}-1\right) \mathcal{S}_{L} w(\widehat{\boldsymbol{x}})$, which is then substituted back in (17) to obtain

$$
\left(\mathcal{S}_{L} \mathcal{S}_{R} w\right)(\widehat{\boldsymbol{x}})=\left(2\|w\|_{R}^{2}-1\right)\left(2\|w\|_{R}^{2} w(\widehat{\boldsymbol{x}})-\left(\mathcal{S}_{R} w\right)(\widehat{\boldsymbol{x}})\right) .
$$

So far, the formulations in the proof are valid for $\widehat{\boldsymbol{x}} \in \mathbb{S}^{2}$. For $\widehat{\boldsymbol{x}} \in R$, we can write (17) as

$$
\left(\mathcal{S}_{L} w\right)(\widehat{\boldsymbol{x}})=\left(2\|w\|_{R}^{2}-1\right) w(\widehat{\boldsymbol{x}}), \quad \widehat{\boldsymbol{x}} \in R,
$$

which, when added to (21), yields (after rearranging terms)

$$
\begin{aligned}
\left(\mathcal{S}_{L} \mathcal{S}_{R} w+\mathcal{S}_{L} w\right)(\widehat{\boldsymbol{x}}) & =2\|w\|_{R}^{2}\left(2\|w\|_{R}^{2}-1\right) w(\widehat{\boldsymbol{x}}), & & \widehat{\boldsymbol{x}} \in R \\
\left(\mathcal{S}_{L} \mathcal{S}_{R} w\right)(\widehat{\boldsymbol{x}}) & =2\|w\|_{R}^{2}\left(2\|w\|_{R}^{2}-1\right) w(\widehat{\boldsymbol{x}}), & \widehat{\boldsymbol{x}} & \in \mathbb{S}^{2} \backslash R .
\end{aligned}
$$

Together with the definition of kernels for the operators $\mathcal{S}_{L}$ and $\mathcal{S}_{R}$, given in (4) and (5), respectively, the above equation implies that $\mathcal{S}_{R L} w(\widehat{\boldsymbol{x}})=\eta w(\widehat{\boldsymbol{x}})$, where the kernel of the operator $\mathcal{S}_{R L}$ is given in (14) and $\eta$ given in (15).

Remark 2. Using (23), it can be shown that each of the basis function $g_{p, k}$, given in (12), is an eigenfunction of the operator $\mathcal{S}_{R L}$, that is, $\left(\mathcal{S}_{R L} g_{p, k}\right)(\widehat{\boldsymbol{x}})=\eta_{p, k} g_{p, k}(\widehat{\boldsymbol{x}})$, where $\eta_{p, k}=$ $\left\|g_{p, k}\right\|_{R}^{2}\left(2\left\|g_{p, k}\right\|_{R}^{2}-1\right)$ as given in (15) serves as a measure of concentration of energy in the spatial and spectral regions of interest.

Remark 3. It can also be proven that the operator $\mathcal{S}_{R L}$ is a projection operator that projects a signal $w \in L^{2}\left(\mathbb{S}^{2}\right)$ to the subspace $\mathcal{H}_{L}+\widetilde{\mathcal{H}}_{R}$. However, this is beyond the scope of current work. Furthermore, the operator $\mathcal{S}_{R L}$ is self-adjoint, by inspection of (14), and shown to be compact [1], and therefore the optimal basis functions are the only eigenfunctions of the operator $\mathcal{S}_{R L}$.

\subsection{Properties and Use of Optimal Basis Functions}

The results presented in the Theorems 1-3 allow us to summarise the following properties of optimal basis functions $g_{p, k}, p \in$ $\left[1, L^{2}\right], k=1,2$.

P1: In addition to being orthonormal over $L^{2}\left(\mathbb{S}^{2}\right)$ or $\mathcal{H}_{L}+\widetilde{\mathcal{H}}_{R}$ (Theorem 2), optimal basis functions $g_{p, k}$ are orthogonal over $\mathcal{H}_{L}$ and $\mathcal{H}_{R}$, that is

$$
\left\langle g_{p, k}, g_{q, k}\right\rangle_{R}=\left\|g_{p, k}\right\|_{R}^{2} \delta_{p, q}, \quad\left\langle g_{p, k}, g_{q, k}\right\rangle_{L}=\left\|g_{p, k}\right\|_{L}^{2} \delta_{p, q} .
$$

P2: Since the eigenvalues are indexed such that $1>\lambda_{p} \geq \lambda_{q}>0$ for $p<q$ and $p, q \in\left[1, L^{2}\right]$, the product of energy concentration measures for optimal basis functions have the following structure (using result from Theorem 1 ) for $p<q$ and $p, q \in\left[1, L^{2}\right]$ :

$$
\begin{aligned}
1 & >\left\|g_{p, 1}\right\|_{R}^{2}\left\|g_{p, 1}\right\|_{L}^{2}>\left\|g_{p, 1}\right\|_{R}^{2}\left\|g_{p, 1}\right\|_{L}^{2}>0.25, \\
0.25 & >\left\|g_{p, 2}\right\|_{R}^{2}\left\|g_{p, 2}\right\|_{L}^{2}>\left\|g_{q, 2}\right\|_{R}^{2}\left\|g_{q, 2}\right\|_{L}^{2}>0 .
\end{aligned}
$$

P3: Following remark 2, the eigenvalue $\eta_{p, k}$ decreases monotonically to zero as $p$ increases from 1 to $L^{2}$ for $k=1$, whereas, it decreases for $k=2$ from a negative value to a minimum and then increases to zero as $p$ increases from 1 to $L^{2}$.

P4: We order the proposed optimal basis $g_{p, k}, p \in\left[1, L^{2}\right], k=1,2$ as $\psi_{1}, \psi_{2}, \ldots, \psi_{2 L^{2}}$, in the order of decreasing magnitude of their eigenvalues. Following Theorem 2 and remark 3, we can project any signal $w \in L^{2}\left(\mathbb{S}^{2}\right)$ to the subspace $\mathcal{H}_{L}+\widetilde{\mathcal{H}}_{R}$ using the proposed optimal basis as follows:

$$
w(\widehat{\boldsymbol{x}})=\sum_{p=1}^{2 L^{2}}\left\langle w, \psi_{p}\right\rangle \psi_{p}(\widehat{\boldsymbol{x}}) .
$$

If the signal $w$ is concentrated in the spatial region $R$ in the spatial domain and in the spectral region $A_{L}$ in the spectral domain, we expect that it can be represented using fewer number of terms as the inner product $\left\langle w, \psi_{p}\right\rangle$ decays to zero as $p$ increases from 1 to $2 L^{2}$.

\section{CONCLUSIONS}

We have designed complete orthonormal basis functions for the joint subspace given by the vector sum of two subspaces formed by band-limited functions and space-limited spectrally concentrated functions. The basis functions are constructed as a linear combination of the band-limited functions, which maximize the energy concentration in some spatial region of interest, and space-limited functions, which maximize the energy concentration in some spectral region of interest. The linear combination of these functions is optimised such that the product of measures of the energy concentration in spatial and spectral domain is maximized. We also show that the optimal basis functions are eigenfunctions of a linear operator which maximizes the product of measures of energy concentration in the spatial and spectral regions of interest. The magnitude of the eigenvalue associated with each eigenfunction quantifies the energy concentration in the spatial and spectral regions of interest. We expect that the proposed basis of simultaneously spatially and spectrally concentrated functions should be useful for signal representation and data analysis in a variety of applications. 


\section{REFERENCES}

[1] R. A. Kennedy and P. Sadeghi, Hilbert Space Methods in Signal Processing. Cambridge, UK: Cambridge University Press, Mar. 2013.

[2] M. K. Chung, K. M. Dalton, L. Shen, A. C. Evans, and R. J. Davidson, "Weighted fourier series representation and its application to quantifying the amount of gray matter," IEEE Trans. Med. Imag., vol. 26, no. 4, pp. 566-581, Apr. 2007.

[3] F. J. Simons, F. A. Dahlen, and M. A. Wieczorek, "Spatiospectral concentration on a sphere," SIAM Rev., vol. 48, no. 3, pp. 504-536, 2006.

[4] Z. Khalid, R. A. Kennedy, S. Durrani, P. Sadeghi, Y. Wiaux, and J. D. McEwen, "Fast directional spatially localized spherical harmonic transform,” IEEE Trans. Signal Process., vol. 61, no. 9, pp. 2192-2203, May 2013.

[5] Z. Khalid, S. Durrani, P. Sadeghi, and R. A. Kennedy, "Spatio-spectral analysis on the sphere using spatially localized spherical harmonics transform," IEEE Trans. Signal Process., vol. 60, no. 3, pp. 1487-1492, Mar. 2012.

[6] Z. Khalid, R. A. Kennedy, and S. Durrani, "On the choice of window for spatial smoothing of spherical data," in Proc. IEEE Int. Conf. Acoustics, Speech and Signal Processing, ICASSP'2014, Florence, Italy, May 2014, pp. 2663-2667.

[7] A. Albertella, F. Sansò, and N. Sneeuw, "Band-limited functions on a bounded spherical domain: the Slepian problem on the sphere," J. Geodesy, vol. 73, no. 9, pp. 436-447, Jun. 1999.

[8] M. A. Wieczorek and F. J. Simons, "Localized spectral analysis on the sphere," Geophys. J. Int., vol. 162, no. 3, pp. 655-675, Sep. 2005.

[9] F. A. Dahlen and F. J. Simons, "Spectral estimation on a sphere in geophysics and cosmology," Geophys. J. Int., vol. 174, pp. 774-807, 2008.

[10] Y. Alem, Z. Khalid, and R. A. Kennedy, "Band-limited extrapolation on the sphere for signal reconstruction in the presence of noise," in Proc. IEEE Int. Conf. Acoustics, Speech and Signal Processing, ICASSP'2014, Florence, Italy, May 2014, pp. 4169-4173.

[11] D. Slepian and H. O. Pollak, "Prolate spheroidal wave functions, Fourier analysis and uncertainity-I," Bell Systems Technical Journal, vol. 40, pp. 43-63, Jan. 1961.

[12] H. J. Landau and H. O.Pollak, "Prolate spheroidal wave functions, Fourier analysis and uncertainity-II," Bell System Tech J., vol. 40, pp. 65-84, Jan. 1961.

[13] D. Slepian and E. Sonnenblick, "Eigenvalues associated with prolate spheroidal wave functions of zero order," Bell System Technical Journal, vol. 44, no. 8, pp. 1745-1759, 1965.

[14] L. Wei, R. A. Kennedy, and T. A. Lamahewa, "Quadratic variational framework for signal design on the 2-sphere," Signal Processing, IEEE Transactions on, vol. 59, no. 11, pp. 52435252, Nov. 2011.

[15] Q. Jin, Z. Luo, and K. M. Wong, "An optimum complete orthonormal basis for signal analysis and design," IEEE Trans. Inf. Theory, vol. 40, no. 3, pp. 732-742, May 1994. 